Check for updates

Cite this: Phys. Chem. Chem. Phys., 2017, 19, 10282

Received 31st December 2016, Accepted 13th March 2017

DOI: 10.1039/c6cp08937b

rsc.li/pccp

\section{Graphene oxide layers modified by light energetic ions}

\author{
Petr Malinský, ${ }^{* a}$ Anna Macková, ${ }^{a b}$ Romana Mikšová, ${ }^{a b}$ Helena Kováčiková, ab \\ Mariapompea Cutroneo, ${ }^{a}$ Jan Luxa, ${ }^{C}$ Daniel Bouša, ${ }^{C}$ Beata Štrochová ${ }^{C}$ and \\ Zdeněk Sofer (iD *c
}

\begin{abstract}
In this paper, the effect of light ion irradiation on graphene oxide foil structure and composition was studied. Due to the excellent properties of graphene based materials suitable for application in electronics, optoelectronics, micro-mechanics and space technologies, the interaction of energetic ions with graphene based structures is worth studying. From the fundamental point of view, it is also interesting to get information about graphene oxide structure modification and the possible functional properties after irradiation by energetic ions. The light ion irradiation of graphene oxide (GO) foil was performed using $2.5 \mathrm{MeV} \mathrm{H}^{+}$and $5.1 \mathrm{MeV} \mathrm{He}^{2+}$ ions. The change in the elemental composition of the GO foils after ion irradiation was investigated using Rutherford Backscattering Spectrometry and Elastic Recoil Detection Analysis. The influence of ion irradiation was further studied by microscopy methods. The chemical composition and structural changes of the GO foil surface were characterized by spectroscopy techniques including XPS, FTIR and Raman spectroscopy. Although the results of ion beam analysis indicated no significant compositional changes in the bulk of GO foils connected to ion irradiation, XPS, ATR-FTIR and Raman spectroscopy revealed reduction and removal of oxygen functionalities on the surface of graphene oxide. This reduction leads to a surface resistivity decrease after ion irradiation dependent on the ion species, fluence and energy.
\end{abstract}

\section{Introduction}

Graphene oxide is well known for several unusual properties with enormous application potential. ${ }^{1-3}$ The main focus of current research concerning graphene oxide material is on purification and separation methods due to its outstanding capability for highly selective water purification. ${ }^{4-6}$ Its perfect mechanical properties and possibilities of further chemical modification open new huge opportunities for flexible electronics, sensors and other devices. ${ }^{3,7}$ Graphene oxide (GO) prepared by oxidation of graphite contains oxygen functionalities, which allow crosslinking of individual sheets by various types of interactions. ${ }^{8}$ Such modification can further improve or control GO properties for application as a membrane material., ${ }^{6,9}$ In addition, the synthesis, electrical and optical properties and application possibilities of graphene oxide and reduced graphene oxide have been extensively studied in recent years. ${ }^{10-12}$

\footnotetext{
${ }^{a}$ Institute of Nuclear Physics AS CR, v.v.i., Husinec - Řež, 130, 25068 Řež, Czech Republic.E-mail: malinsky@ujf.cas.cz

${ }^{b}$ Department of Physics, Faculty of Science, J.E. Purkinje University, Ceske Mladeze 8, 40096 Usti nad Labem, Czech Republic

${ }^{c}$ Department of Inorganic Chemistry, University of Chemistry and Technology Prague,

Technická 5, 16628 Prague 6, Czech Republic. E-mail: zdenek.sofer@vscht.cz
}

Numerous experiments have demonstrated that various types of defects can be efficiently introduced into the graphene structure using irradiation with energetic particles such as ions. ${ }^{13-15}$ High density ion fluence irradiation with a focused ion beam can be used for cutting and patterning of graphene with a high spatial resolution. ${ }^{16}$ The results indicate that understanding the defect production in graphene and graphene based materials under ion bombardment is mandatory for successful treatment of graphene by ion beams. ${ }^{17}$ The light energetic ion interaction with graphene based structures is in the focus of space technologies, where the deterioration of device performance via light energetic particles is expected. The great significance of graphene-based devices is in space application, ${ }^{18}$ particularly in graphene solar cells, ${ }^{19}$ and the stability of these devices in the harsh environment is best studied by the interaction of graphene with ions of $\mathrm{MeV}$ energies. ${ }^{20-22}$ Graphene allotropes, including graphene oxide, are interesting materials with excellent electronic, ${ }^{23}$ mechanical ${ }^{24}$ and thermal properties. $^{25}$ The ion irradiation and/or ion implantation of graphene and graphene oxide is rarely mentioned in the literature and the possible functional optical and/or electrical properties of graphene based structures modified by the energetic ion are still unknown. Our work is focused on light energetic ion interaction with graphene oxide foils as a base for further functionalizing graphene oxide using ion beams. 
In the present work the modification of graphene oxide foils by $\mathrm{H}^{+}$and $\mathrm{He}^{2+}$ ion irradiation was investigated with the reference ion energies of 2.5 and $5.1 \mathrm{MeV}$, respectively. The ions and the energies were selected according to previously realized experiments determining the energy stopping power and straggling in graphene oxide (GO) foils. ${ }^{26}$ In this work, the structural and compositional changes of the irradiated GO foils are discussed in detail.

\section{Experimental}

\section{Preparation of the graphene oxide foil}

The present experiment used graphene oxide (GO) foil prepared by graphite oxidation utilising the permanganate oxidation method. ${ }^{27} 3 \mathrm{~g}$ of graphite $(2-15 \mu \mathrm{m}, 99.9995 \%$, Alfa Aesar) was mixed with $360 \mathrm{~mL}$ of $\mathrm{H}_{2} \mathrm{SO}_{4}\left(96 \mathrm{wt} \%\right.$ ) and $40 \mathrm{~mL}$ of $\mathrm{H}_{3} \mathrm{PO}_{4}$ (85 wt\%). Subsequently, $18 \mathrm{~g}$ of $\mathrm{KMnO}_{4}$ was added and the reaction mixture was heated to $50{ }^{\circ} \mathrm{C}$ for 12 hours. Afterwards, the reaction mixture was quenched in ice $(400 \mathrm{~g})$ with $20 \mathrm{~mL}$ of hydrogen peroxide (30 wt\%), and the formed graphene oxide was separated by centrifugation. GO foils were prepared by suction filtration using a polycarbonate membrane (Nucleopore $0.45 \mu \mathrm{m}$ ) and graphene oxide suspension. The GO foils were prepared using an aqueous suspension with a concentration of $6.7 \mathrm{mg} \mathrm{mL} \mathrm{m}^{-1}$. The GO foils were stored in a paper wrapper to prevent reduction due to degradation by visible light. ${ }^{28}$

The mass density of graphene oxide foil $\left(1.36 \pm 0.06 \mathrm{~g} \mathrm{~cm}^{-3}\right)$ was determined by the standard technique of microbalance weighing by means of a Mettler Toledo Micro-Balance with $\pm 1 \mu \mathrm{g}$ absolute accuracy. A piece of GO foil was cut and the area of this piece was precisely determined using image analysis from a digital microscope image. The thickness of the GO foil was measured using an optical microscope on the cut through the graphene oxide foil that was clamped between two polymer cubes by drenched resin. The cut surface was polished perpendicular to the surface of the GO foil (see Fig. 1). The final thickness was determined to be $50 \pm 2 \mu \mathrm{m}$.

The GO foils were irradiated with $2.5 \mathrm{MeV} \mathrm{H}^{+}$and $5.1 \mathrm{MeV}$ $\mathrm{He}^{2+}$ ions in the implantation chamber installed at the $3 \mathrm{MV}$ Tandetron MC 4130 accelerator. The ion irradiation fluencies used were $1.0 \times 10^{13} \mathrm{~cm}^{-2}, 1.0 \times 10^{14} \mathrm{~cm}^{-2}$ and $1.0 \times$ $10^{15} \mathrm{~cm}^{-2}$ for both types of ions. The ion irradiation was provided using an ion current density of 7.5-15.4 $\mathrm{nA} \mathrm{cm}^{-2}$ in a vacuum of about $6.9 \times 10^{-6}$ mbar.

\section{Compositional study using RBS and ERDA}

Rutherford Backscattering Spectrometry (RBS) and Elastic Recoil Detection Analysis (ERDA) were employed for the compositional study of GO foil before and after ion irradiation. The RBS spectra were measured using a beam of $2.0 \mathrm{MeV} \mathrm{He}^{+}$ions. An Ultra-Ortec PIPS detector recorded $\mathrm{He}^{+}$ions backscattered at a laboratory scattering angle of $170^{\circ}$. The ERDA spectra were measured using 2.5 $\mathrm{MeV} \mathrm{He}^{+}$ions. The primary beam was incident at an angle of $75^{\circ}$ with respect to the foil surface normal, and hydrogen atoms that recoiled at a scattering angle of $30^{\circ}$ were registered using a

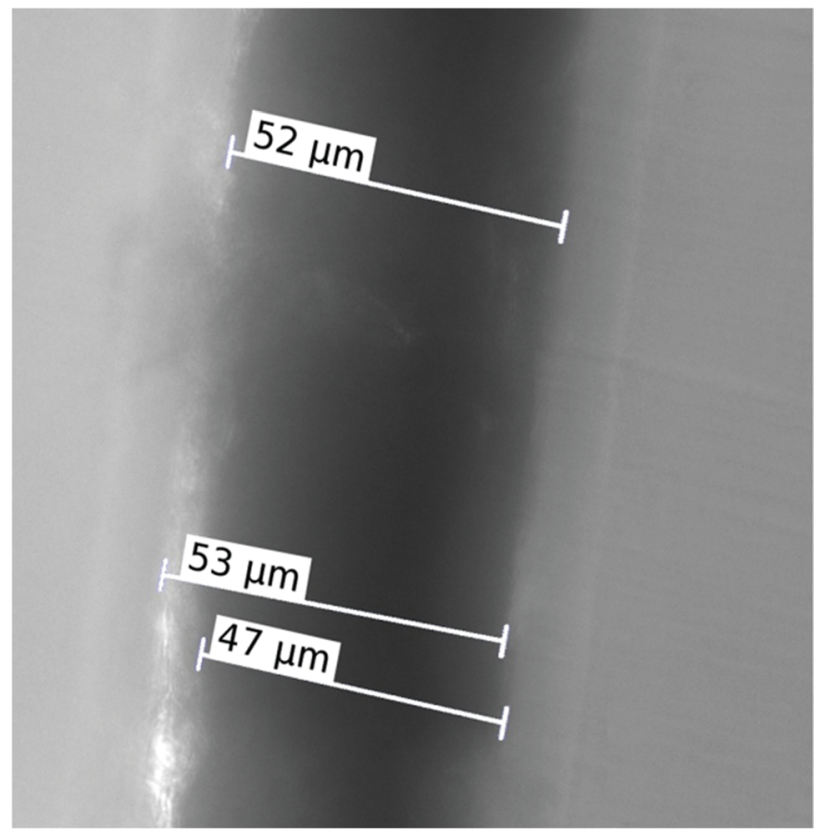

Fig. 1 Optical microscope image of the graphene oxide foil cut.

detector covered by a $12 \mu \mathrm{m}$ Mylar foil. The typical ion current used during RBS and ERDA analysis was $5 \mathrm{nA}$. To reduce the effects of sample degradation during RBS/ERDA analysis, several particular spectra were measured on different beam spots and the final spectrum was obtained by summing the individual spectra. RBS and ERDA spectra were evaluated using the SIMNRA code. ${ }^{29}$ RBS and ERDA give us information about the compositional changes in the surface layer, where mostly electronic stopping takes place, and electronic stopping is a prevailing phenomenon in energy loss (see also Fig. 2, where SRIM simulation of the investigated ion penetration through GO foil is presented). Simultaneously, the precise prediction of the ion energy transfer into the graphene oxide foils can be realized only in the case of known chemical composition and density before ion irradiation. ${ }^{26}$

\section{GO surface morphology determined by AFM measurements}

The surface morphology changes were followed as they can provide information about the structural changes of the surface of the irradiated material and can be discussed in the frame of elemental and structural changes after ion irradiation.

AFM morphological study and conductive AFM (C-AFM) of graphene foils were done before and after irradiation. The surface morphology, roughness and $I-V$ characteristics were examined by Atomic Force Microscopy (AFM). The AFM NTEGRA Spectra from NT-MDT were measured in a tapping mode. The average roughness $\left(R_{\mathrm{a}}\right)$ and the root mean square (RMS) of the profile height deviations from the centre plane were calculated. The $I-V$ characteristics were measured in the voltage range of $-4 \mathrm{~V}$ to $+4 \mathrm{~V}$.

\section{Optical and electrical properties of the irradiated GO foils}

The changes of the electrical properties are related to structural changes in the irradiated GO foils. The electronic structure modification and bond rearrangement are prevailingly connected 

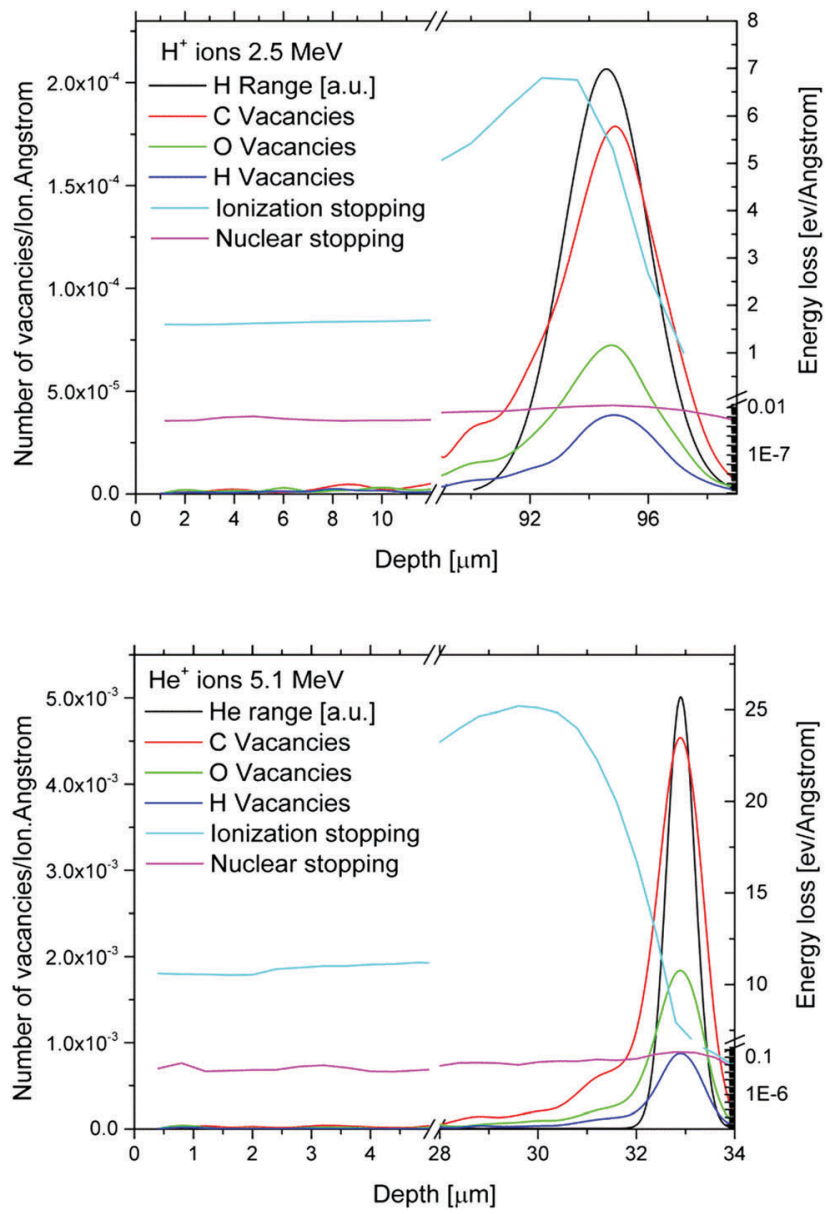

Fig. 2 SRIM simulation of the electronic and nuclear energy stopping and vacancies in $\mathrm{GO}$ created by ion irradiation are presented for $2.5 \mathrm{MeV} \mathrm{H}^{+}$ (up) and $5.1 \mathrm{MeV} \mathrm{He}^{+}$ions (down) simultaneously with $\mathrm{H}^{+}$and $\mathrm{He}^{+}$ion depth distribution.

to the electronic stopping of energetic light ions in matter which prevails in the ion energy range used. These electronic structure modifications can significantly change the electrical properties of irradiated foils. Focused energetic ion beams can be used for micro-patterning of polymers, which is highly desirable in the case of graphene based structures to create microstructures with modified electrical resistivity. Thus, the study of electric resistivity of the irradiated GO foil is an important issue. In the same way, optical properties are closely connected to structural changes and electrical properties, and thus we also carried out an analysis of the optical properties of the ion irradiated GO foils in this work.

The current-voltage $(I-V)$ characteristics of pristine and irradiated GO foils were studied by the standard 2-point method utilizing a Keithley 6221 current source and a Keithley 2128A nano-voltmeter. The $\mathrm{Ag}$ contacts (50 nm thick) were sputtered on the surface of GO foils for electrical resistance measurements.

The optical properties of virgin and implanted GO foils were measured in a reflection mode, to cover the $\mathrm{UV}$, visible and near-IR regions of the spectrum, using an AvaSpec-2048 spectrophotometer with UB-600 lines/mm grating and a bandwidth from $195 \mathrm{~nm}$ to $757 \mathrm{~nm}$. A dual light source from the Avantes line of instruments, consisting of halogen/deuterium lamps, was employed in the range of 175-2500 $\mathrm{nm}$ and angle of incidence at $15^{\circ}$. The light impinged on the sample at an incidence angle of $-15^{\circ}$ with respect to the normal to the target plane and the optical spectrometer detected at $+15^{\circ}$.

\section{Structural and composition modification of GO foils determined using XPS, FTIR and Raman spectroscopy}

Raman spectroscopy, ATR-FTIR and XPS measurements were used to get information about the structural and elemental modification of the surface of GO foils caused by energetic ion irradiation. Raman spectroscopy is considered as a powerful method to study carbon based materials due to the specific response to changes in the carbon hybridization state and introduction of defects. ${ }^{30}$ FTIR spectroscopy is nowadays considered as one of the most important tools to characterise chemical bonding within the carbon film since the involved groups and formed covalent bonds have characteristic absorption bands in the infrared spectra. ${ }^{31}$ XPS offers information about the chemical bonds and elemental composition in the very shallow subsurface region of the investigated graphene oxide foil under the ion beam.

An inVia Raman microscope (Renishaw, England) was used for Raman spectroscopy measurements. The spectrometer operates in backscattering geometry with a CCD detector. An Nd-YAG laser $(532 \mathrm{~nm}, 50 \mathrm{~mW})$ with a $50 \times$ magnification objective was used for measurements. Instrument calibration was achieved with a silicon reference which yields a peak position at $520 \mathrm{~cm}^{-1}$. To avoid sample damage, no more than $5 \%$ of the total $50 \mathrm{~mW}$ laser power was used. Samples were drop-cast on a silicon wafer from an isopropanol suspension $\left(1 \mathrm{mg} \mathrm{mL}{ }^{-1}\right)$ in order to perform the measurements.

Attenuated total reflectance Fourier transform infrared spectroscopy (ATR-FTIR) measurements were performed on a NICOLET iS50R FTIR spectrometer (Thermo Scientific, USA). A Diamond ATR crystal and a DTGS detector were used for all measurements, which were carried out in the range of 4000$400 \mathrm{~cm}^{-1}$ at a resolution of $4 \mathrm{~cm}^{-1}$.

High resolution X-ray photoelectron spectroscopy (XPS) was performed with an ESCAProbeP (Omicron Nanotechnology Ltd, Germany) spectrometer using a monochromatic aluminium X-ray radiation source (1486.7 eV). A wide-scan survey of all elements was performed, with subsequent high-resolution scans of the $\mathrm{C}$ 1s and $\mathrm{O}$ 1s core level spectra. For the evaluation of the carbon-to-oxygen (C/O) ratios from the survey spectra, relative sensitivity factors were used. Prior to measurement, samples were applied onto conductive carbon tape. To eliminate sample charging during measurement (1-5 V) an electron gun was used and the acquisition time of all XPS measurements was reduced to minimize the possibility of surface damage by X-rays. ${ }^{32}$

\section{Results}

\section{Simulations of the structural changes after ion irradiation}

For the initial estimation of the defect evolution caused by $\mathrm{H}^{+}$ and $\mathrm{He}^{2+}$ ion irradiation in the graphene oxide foils, the SRIM 
$\operatorname{code}^{33}$ was employed. When a beam of charged particles penetrates matter, ion deceleration is accompanied by electronic and nuclear stopping. The interplay of these two phenomena is crucial for structural changes of irradiated substrates. Electronic stopping (the prevailing phenomenon in our case) generally leads to atom ionisation, electron excitation and subsequent creation of free radicals and free chemical bonds. Nuclear stopping leads to atom knocking and production of vacancies. ${ }^{33}$ The SRIM package simulates the depth profiles of irradiation induced vacancy-interstitial Frenkel pairs (FP) using the binary collision approximation method and is unable to include the charge state fluctuation of projectile ions. In Fig. 2 we can see the depth profiles of the oxygen, hydrogen and carbon created vacancies in GO foil (using the full cascade MC simulation by SRIM) for $\mathrm{He}^{+}$and $\mathrm{H}^{+}$ions based on the chemical composition obtained from RBS. Simultaneously, the ion projected ranges realized at the above mentioned ion irradiation parameters are presented.

In our case, the ratio of electronic stopping to nuclear stopping $\left(S_{\mathrm{e}} / S_{\mathrm{n}}\right)$ was 1720 for $\mathrm{H}^{+}$ions with energy $2.5 \mathrm{MeV}$ and 1500 for $\mathrm{He}^{+}$ions with energy $5.1 \mathrm{MeV}$. The simulated projected range for $\mathrm{H}^{+}$ions was $94.5 \mu \mathrm{m}$ and for $\mathrm{He}^{+}$ions $32.9 \mu \mathrm{m}$. It is evident that almost all $\mathrm{H}^{+}$ions passed through the irradiated GO foil and that the $\mathrm{He}^{2+}$ ions are stopped deep inside the graphene oxide. The analytical techniques used were performed in such a way that they characterized the surface layer influenced exceptionally by ion electronic losses which is the focus of our research. The sum of SRIM predicted $\mathrm{C}, \mathrm{H}$ and $\mathrm{O}$ vacancies created in the investigated GO foil by $5.1 \mathrm{MeV} \mathrm{He}^{+}$ions is 5.9 times higher compared to the sum of all vacancies created by $2.5 \mathrm{MeV}$ $\mathrm{H}^{+}$ions.

\section{Compositional study using RBS and ERDA}

The elemental compositions of GO foil before and after ion irradiation for all used ion fluencies evaluated using RBS and ERDA are presented in Table 1 . The accessible information depth in the case of graphene oxide and $2 \mathrm{MeV} \mathrm{He}^{+}$ions, used as a probe in RBS analysis, is about $1 \mu \mathrm{m}$. Except for the presumed majority elements - carbon, hydrogen and oxygen, additional elements such as sulphur and manganese are also present in the analysed GO foils which originate from the synthesis procedure used for the preparation of GO foils. Hydrogen is generally present in graphene oxide in the form of hydroxyl functional groups, in which the hydrogen/oxygen $(\mathrm{H} / \mathrm{O})$ ratio typically reaches a value of 1 , and the ratio $\mathrm{H} / \mathrm{O}$ of about 0.5 is ascribed to carboxylic acids and 0 for ketones and epoxides. ${ }^{27}$ In our case the hydrogen/oxygen ratio is in the range of $0.38-0.48$, and thus we can conclude the presence of carboxylic groups. The highest $\mathrm{H} / \mathrm{O}$ ratio (0.48) was observed for pristine GO foil and this ratio decreases after ion irradiation, and thus the reduction of hydroxyl and carboxyl functional groups is suggested, which is further confirmed by XPS and ATR-FTIR analysis. It is obvious from Table 1 that after ion irradiation the elemental composition of GO foils, analysed using RBS and ERDA methods, exhibits only small changes as the electronic stopping prevailingly modified the structure and no significant escape of elements is observable because nuclear stopping is significantly less for both ions used as was predicted by SRIM simulation (see Fig. 2). Negligible elemental changes were expected, as the high energy light ions prevailingly modify the electronic structure of the irradiated material, which in consequence is exhibited in optical and electrical property modification.

\section{GO surface morphology determined by AFM measurements}

The GO foil morphology was investigated using Atomic Force Microscopy (AFM) in the tapping mode. AFM images and the corresponding surface height profiles before and after ion irradiation are shown in Fig. 3. All samples showed a very rough and irregular surface with a maximal height of the profile on the order of $\mu \mathrm{m}$. It can be seen that after ion irradiation, the height of grains decreases from $1.6 \mu \mathrm{m}$ for pristine GO foil to $0.8 \mu \mathrm{m}$ for irradiated GO foil with an ion irradiation fluence of $1.0 \times$ $10^{15} \mathrm{~cm}^{-1}$. The narrowing of the valleys on the GO surface can also be observed with increasing ion fluence for both ion species used. The main roughness characteristics are summarized in Table 2 . The average roughness $\left(R_{\mathrm{a}}\right)$ and root mean square (RMS) decrease with increasing ion fluence for $2.5 \mathrm{MeV} \mathrm{H}^{+}$ions (see Table 2). In the case of $\mathrm{He}^{+}$ion irradiated GO foils, no such dependence is observed. The similar phenomenon was observed in the literature, where GO surface roughness decline under laser beam irradiation was presented. ${ }^{34}$

\section{Structural and composition modification of GO foils determined using XPS, FTIR and Raman spectroscopy}

The elemental species and chemical states in the surface of the GO foils before and after ion beam irradiation were investigated by means of X-ray Photoelectron Spectroscopy (XPS). This technique usually provides information about the sample surface, revealing details within several nanometres. The quantification of

Table 1 The elemental composition of GO foils determined by RBS and ERDA

Composition of the deposited foils [at\%]

\begin{tabular}{|c|c|c|c|c|c|}
\hline \multirow{2}{*}{ Sample/ions } & & & & & \\
\hline & $\mathrm{C}$ & $\mathrm{O}$ & $\mathrm{H}$ & $\mathrm{C} / \mathrm{O}$ & $\mathrm{H} / \mathrm{O}$ \\
\hline Pristine GO foil & $63.5 \pm 1.6$ & $23.7 \pm 0.5$ & $11.4 \pm 0.6$ & 2.68 & 0.48 \\
\hline $5.1 \mathrm{MeV} \mathrm{He}^{2+} 1.0 \times 10^{13} \mathrm{~cm}^{-2}$ & $64.5 \pm 1.6$ & $23.7 \pm 0.5$ & $10.0 \pm 0.5$ & 2.72 & 0.42 \\
\hline $5.1 \mathrm{MeV} \mathrm{He} e^{2+} 1.0 \times 10^{14} \mathrm{~cm}^{-2}$ & $64.2 \pm 1.6$ & $24.5 \pm 0.6$ & $10.3 \pm 0.5$ & 2.62 & 0.42 \\
\hline $5.1 \mathrm{MeV} \mathrm{He}{ }^{2+} 1.0 \times 10^{15} \mathrm{~cm}^{-2}$ & $64.0 \pm 1.6$ & $24.7 \pm 0.6$ & $10.1 \pm 0.5$ & 2.59 & 0.41 \\
\hline $2.5 \mathrm{MeV} \mathrm{H}^{+} 1.0 \times 10^{13} \mathrm{~cm}^{-2}$ & $65.3 \pm 1.6$ & $23.0 \pm 0.5$ & $10.3 \pm 0.5$ & 2.84 & 0.45 \\
\hline $2.5 \mathrm{MeV} \mathrm{H}^{+} 1.0 \times 10^{14} \mathrm{~cm}^{-2}$ & $63.7 \pm 1.5$ & $24.8 \pm 0.6$ & $10.2 \pm 0.5$ & 2.57 & 0.41 \\
\hline $2.5 \mathrm{MeV} \mathrm{H}^{+} 1.0 \times 10^{15} \mathrm{~cm}^{-2}$ & $65.7 \pm 1.6$ & $23.2 \pm 0.5$ & $9.0 \pm 0.5$ & 2.83 & 0.38 \\
\hline
\end{tabular}



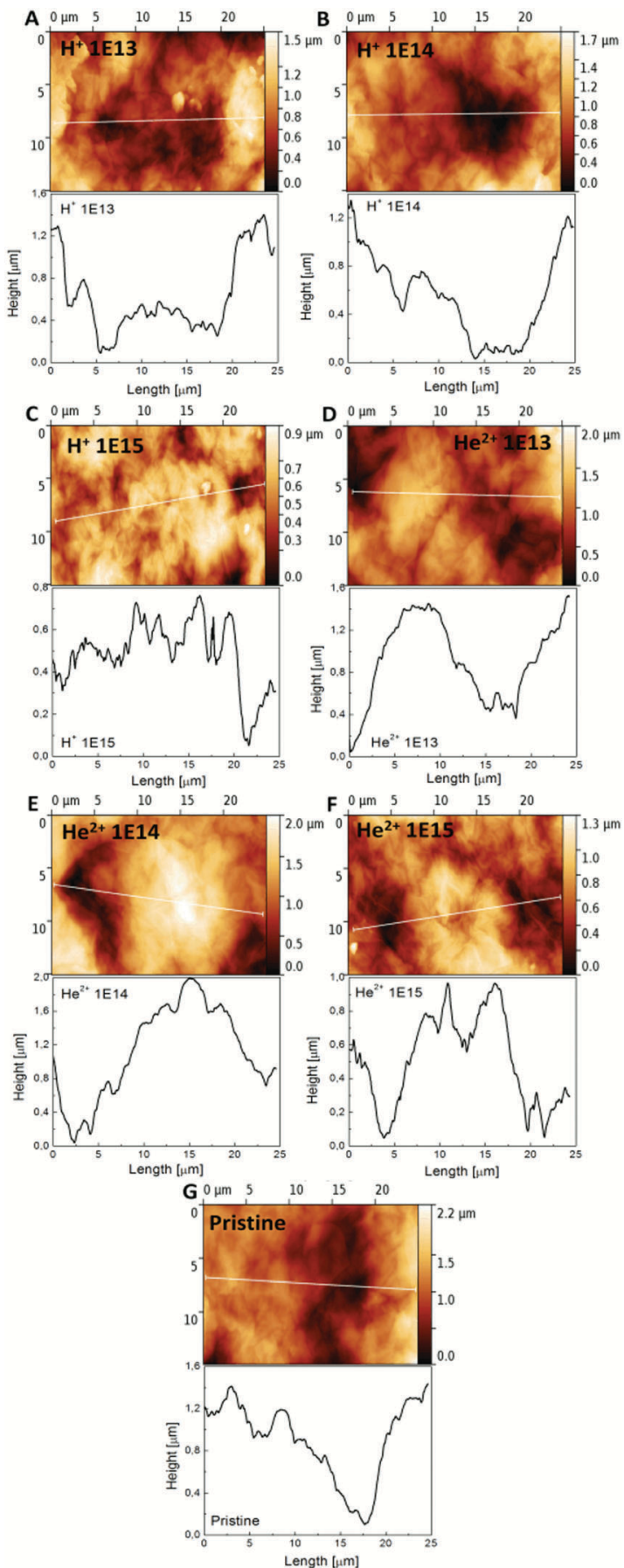

Fig. 3 The AFM images of graphene oxide foils before and after ion irradiation: (A) GO foil irradiated using $2.5 \mathrm{MeV} \mathrm{H}^{+}, 1.0 \times 10^{13} \mathrm{~cm}^{-2}$, (B) GO foil irradiated using $2.5 \mathrm{MeV} \mathrm{H}^{+}, 1.0 \times 10^{14} \mathrm{~cm}^{-2}$, (C) GO foil irradiated using $2.5 \mathrm{MeV} \mathrm{H}^{+}, 1.0 \times 10^{15} \mathrm{~cm}^{-2}$, (D) GO foil irradiated using $5.1 \mathrm{MeV} \mathrm{He}^{2+}, 1.0 \times 10^{13} \mathrm{~cm}^{-2}$, (E) GO foil irradiated using $5.1 \mathrm{MeV} \mathrm{He}^{2+}$, $1.0 \times 10^{14} \mathrm{~cm}^{-2}$, (F) GO foil irradiated using $5.1 \mathrm{MeV} \mathrm{He}^{2+}, 1.0 \times 10^{15} \mathrm{~cm}^{-2}$ and $(\mathrm{G})$ pristine $\mathrm{GO}$ foil.

the $\mathrm{C} 1 \mathrm{~s}$ and $\mathrm{O}$ 1s peaks and the ratio of $\mathrm{C} / \mathrm{O}$ obtained from XPS spectra are summarized in Table 3 . The carbon concentration and the $\mathrm{C} / \mathrm{O}$ ratio are significantly enhanced for the ion fluence $1.0 \times 10^{15} \mathrm{~cm}^{-2}$ for both ion species. Even though the increase in the $\mathrm{C} / \mathrm{O}$ ratio is low, it is shown further in this work that this
Table 2 The roughness parameters $R_{\mathrm{a}}$ and RMS for pristine and irradiated GO foils

\begin{tabular}{|c|c|c|}
\hline Sample & $R_{\mathrm{a}}[\mathrm{nm}]$ & $\mathrm{RMS}[\mathrm{nm}]$ \\
\hline Pristine GO foil & 290 & 357 \\
\hline $2.5 \mathrm{MeV} \mathrm{H}^{+} 1.0 \times 10^{13} \mathrm{~cm}^{-2}$ & 242 & 292 \\
\hline $2.5 \mathrm{MeV} \mathrm{H}^{+} 1.0 \times 10^{14} \mathrm{~cm}^{-2}$ & 236 & 297 \\
\hline $2.5 \mathrm{MeV} \mathrm{H}^{+} 1.0 \times 10^{15} \mathrm{~cm}^{-2}$ & 122 & 150 \\
\hline $5.1 \mathrm{MeV} \mathrm{He}^{2+} 1.0 \times 10^{13} \mathrm{~cm}^{-2}$ & 284 & 345 \\
\hline $5.1 \mathrm{MeV} \mathrm{He}^{2+} 1.0 \times 10^{14} \mathrm{~cm}^{-2}$ & 358 & 432 \\
\hline $5.1 \mathrm{MeV} \mathrm{He}^{2+} 1.0 \times 10^{15} \mathrm{~cm}^{-2}$ & 194 & 233 \\
\hline
\end{tabular}

Table 3 The $\mathrm{C} / \mathrm{O}$ ratio determined from XPS spectra for pristine GO and $\mathrm{GO}$ irradiated using $2.5 \mathrm{MeV} \mathrm{H}^{+}$and $5.1 \mathrm{MeV} \mathrm{He}^{2+}$ ions with different ion irradiation fluencies

\begin{tabular}{llll}
\hline Sample & C 1s (at\%) & O 1s (at\%) & C/O ratio \\
\hline Pristine GO foil & 71.8 & 28.2 & 2.55 \\
$2.5 \mathrm{MeV} \mathrm{H}^{+} 1.0 \times 10^{13} \mathrm{~cm}^{-2}$ & 68.9 & 31.1 & 2.15 \\
$2.5 \mathrm{MeV} \mathrm{H}^{+} 1.0 \times 10^{14} \mathrm{~cm}^{-2}$ & 70.3 & 29.7 & 2.37 \\
$2.5 \mathrm{MeV} \mathrm{H}^{+} 1.0 \times 10^{15} \mathrm{~cm}^{-2}$ & 75.2 & 24.8 & 3.03 \\
$5.1 \mathrm{MeV} \mathrm{He}^{2+} 1.0 \times 10^{13} \mathrm{~cm}^{-2}$ & 70.7 & 29.3 & 2.41 \\
$5.1 \mathrm{MeV} \mathrm{He}^{2+} 1.0 \times 10^{14} \mathrm{~cm}^{-2}$ & 71.0 & 29.0 & 2.45 \\
$5.1 \mathrm{MeV} \mathrm{He}^{2+} 1.0 \times 10^{15} \mathrm{~cm}^{-2}$ & 78.5 & 21.5 & 3.65 \\
\hline
\end{tabular}

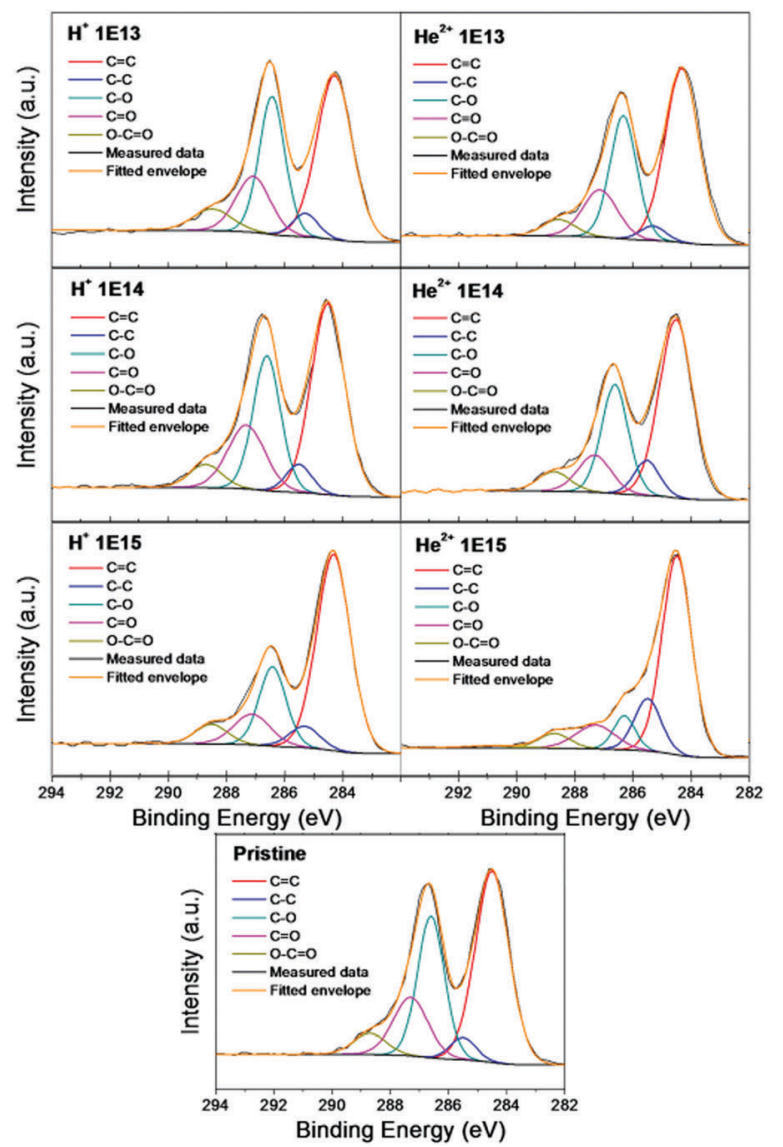

Fig. 4 The deconvolution of the XPS C 1s peak of pristine GO foil and GO foils irradiated using $2.5 \mathrm{MeV} \mathrm{H}^{+}$and $5.1 \mathrm{MeV} \mathrm{He}^{2+}$ ions using various ion fluencies. 
Table 4 Quantitative comparison of carbon bonding states manifested by differences in C 1s binding energies obtained from high resolution XPS spectra

\begin{tabular}{|c|c|c|c|c|c|}
\hline Sample & $\mathrm{C}=\mathrm{C}$ & $\mathrm{C}-\mathrm{C}$ & $\mathrm{C}-\mathrm{O}$ & $\mathrm{C}=\mathrm{O}$ & $\mathrm{O}-\mathrm{C}=\mathrm{O}$ \\
\hline $2.5 \mathrm{MeV} \mathrm{H}^{+} 1.0 \times 10^{13} \mathrm{~cm}^{-2}$ & $45.8 \pm 1.1$ & $4.7 \pm 0.1$ & $27.8 \pm 0.6$ & $15.0 \pm 0.3$ & $6.7 \pm 0.2$ \\
\hline $2.5 \mathrm{MeV} \mathrm{H}^{+} 1.0 \times 10^{14} \mathrm{~cm}^{-2}$ & $46.1 \pm 1.1$ & $5.2 \pm 0.1$ & $26.4 \pm 0.6$ & $17.0 \pm 0.4$ & $5.3 \pm 0.1$ \\
\hline $5.1 \mathrm{MeV} \mathrm{He} \mathrm{H}^{2+} 1.0 \times 10^{13} \mathrm{~cm}^{-2}$ & $50.3 \pm 1.2$ & $3.1 \pm 0.1$ & $28.0 \pm 0.6$ & $14.0 \pm 0.3$ & $4.6 \pm 0.1$ \\
\hline $5.1 \mathrm{MeV} \mathrm{He}{ }^{2+} 1.0 \times 10^{14} \mathrm{~cm}^{-2}$ & $51.5 \pm 1.2$ & $7.6 \pm 0.2$ & $24.9 \pm 0.6$ & $10.5 \pm 1.3$ & $5.5 \pm 0.1$ \\
\hline $5.1 \mathrm{MeV} \mathrm{He}^{2+} 1.0 \times 10^{15} \mathrm{~cm}^{-2}$ & $62.2 \pm 1.4$ & $15.0 \pm 0.3$ & $7.8 \pm 0.2$ & $10.1 \pm 0.2$ & $4.9 \pm 0.1$ \\
\hline
\end{tabular}

enhancement is sufficient in order to significantly increase the conductivity of the GO foil as discussed later on. RBS analysis shows the slight decline of the $\mathrm{C} / \mathrm{O}$ ratio, but it must be emphasized that XPS provides information only about the close subsurface layer about several nm while RBS is able to evaluate the elemental composition averaged over the thick layers according to the penetration depth of the ion probe beam, e.g. about several micrometres. It can be assumed that the XPS observed $\mathrm{C} / \mathrm{O}$ ratio is higher for the fluence $1.0 \times 10^{15} \mathrm{~cm}^{-2}$ compared to RBS analysis due to the slightly higher surface decomposition of oxygen functionalities, as was observed after other type of ionizing irradiation - laser irradiation. ${ }^{34,35}$

The deconvolution of the high resolution XPS spectra of the $\mathrm{C}$ 1s peak of pristine and irradiated GO foils is shown in Fig. 4 and Table 4. In this figure, one can indicate the presence of five different carbon bonding states; $\mathrm{C}=\mathrm{C}$ (284.4), $\mathrm{C}-\mathrm{C}$ (285.4), $\mathrm{C}-\mathrm{O}$ (286.3), $\mathrm{C}=\mathrm{O}(288.0)$ and $\mathrm{O}-\mathrm{C}=\mathrm{O}$ (289.0). Among these bonding states, $\mathrm{C}=\mathrm{C}$ and $\mathrm{C}-\mathrm{O}$ dominated in pristine $\mathrm{GO}$ foil and indicate the considerable degree of oxidation. ${ }^{36}$ After ion irradiation, a decrease of peak intensity is observed, attributed to the oxygen functionalized carbon and increasing intensity of the $\mathrm{C}=\mathrm{C}$ and $\mathrm{C}-\mathrm{C}$ peaks. Although all five indicated carbon chemical states are present for all the GO foils, the concentration of individual chemical states varies. The $\mathrm{C}=\mathrm{C}$ peak gradually becomes more dominant for GO foils irradiated using higher ion fluence, reaching a maximum for GO foil irradiated using $5.1 \mathrm{MeV} \mathrm{He}^{+}$ions, $1.0 \times 10^{15} \mathrm{~cm}^{-2}$. This phenomenon demonstrates the removal of the oxygen containing groups and creation of the carbon groups with increasing ion fluence. Similar results were observed when GO was irradiated using $100 \mathrm{MeV} \mathrm{Au}$ ions in ref. 36 and using Ti ions with different energies. $^{37}$

The ATR-FTIR spectra of the pristine and irradiated GO foils recorded in the wavenumber range of $4000-400 \mathrm{~cm}^{-1}$ are presented in Fig. 5. The pristine GO shows characteristic bands that confirm the presence of functional groups, such as $\mathrm{O}-\mathrm{H}$, $\mathrm{C}=\mathrm{O}, \mathrm{C}-\mathrm{OH}$, and $\mathrm{C}-\mathrm{O}$. The broad characteristic peak between 3000 and $3600 \mathrm{~cm}^{-1}$ represents the $\mathrm{O}-\mathrm{H}$ stretching of hydroxyl and carboxyl groups which is significantly reduced with increasing ion irradiation fluence for $\mathrm{He}^{2+}$ and $\mathrm{H}^{+}$ions. ${ }^{38,39}$ The oxygen in the pristine sample can be confirmed mainly by the absorption bands at $\sim 1730 \mathrm{~cm}^{-1}$ ( $\mathrm{C}=\mathrm{O}$ stretching vibration) and by the presence of a broad band at $1000-1200 \mathrm{~cm}^{-1}$ attributed to $\mathrm{C}-\mathrm{O}$ alkoxy and epoxy stretching vibration. ${ }^{36,40,41}$ The presence of the absorption peak at $\sim 1630 \mathrm{~cm}^{-1}$ can be attributed to the stretching vibration of $\mathrm{C}=\mathrm{C}$ of the graphene skeleton. ${ }^{38}$ The presence of hydroxyl groups results in the formation of hydrogen bonds which contribute to the hydrophilic nature of graphene oxide. Fig. 5 shows that with increasing ion irradiation fluence, the intensity of all the peaks ascribed to the oxygen chemical states decrease and this decrease is more pronounced for GO samples irradiated using $\mathrm{He}^{2+}$ ions. The ATR-FTIR spectra indicated the chemical reduction of GO foil after ion irradiation due to the electronic energy transferred from $\mathrm{H}^{+}$and $\mathrm{He}^{2+}$ ions to the GO system and the GO reduction is more pronounced for $\mathrm{He}^{2+}$ ions as predicted by SRIM simulation as the ionization of GO is to a larger extent realized by $\mathrm{He}^{+}$ions compared to $\mathrm{H}^{+}$ions. The chemical
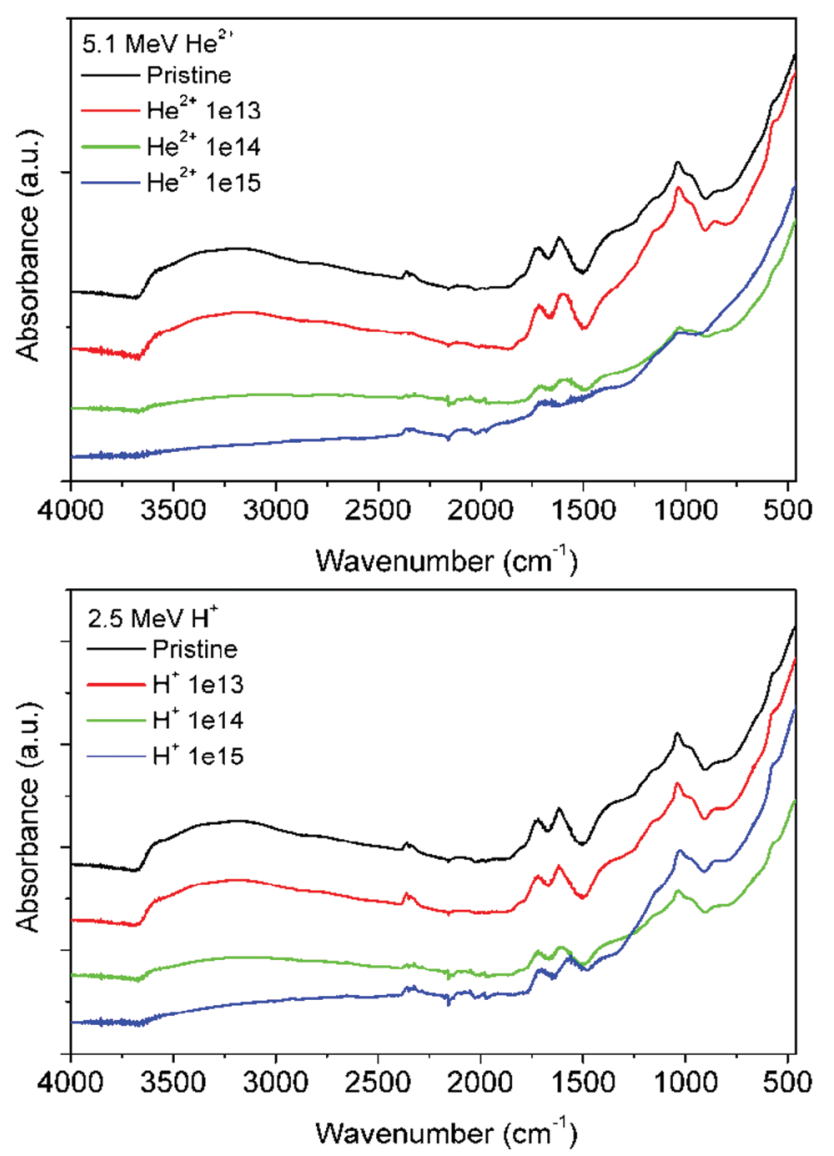

Fig. 5 The ATR-FTIR spectra of pristine and irradiated GO foils. The ion beam irradiation was performed using $2.5 \mathrm{MeV} \mathrm{H}^{+}$and $5.1 \mathrm{MeV} \mathrm{He}^{2+}$ ions with different fluencies. 
reduction of GO was accompanied by a decrease or even suppression of the intensity of different oxygen peaks as observed in FTIR spectra. $^{36}$

To get more comprehensive insight into graphene oxide reduction under the light energetic ion irradiation, Raman spectroscopy was used. In all Raman spectra, two major peaks, corresponding to the $\mathrm{D}\left(1350 \mathrm{~cm}^{-1}\right)$ and $\mathrm{G}\left(1590 \mathrm{~cm}^{-1}\right)$ bands, were observed (see Fig. 6). The D band is assigned to defect induced Raman features, and this band cannot be seen for highly crystalline graphite/graphene samples. ${ }^{42}$ The $\mathrm{G}$ band peak is associated with the vibration of $\mathrm{sp}^{2}$ bonded carbon atoms in graphene. ${ }^{2,41,43}$ The double peak present around $2700 \mathrm{~cm}^{-1}$ is generally labelled as $2 \mathrm{D}$ band and indicates the reduction of GO. ${ }^{41}$ The intensity ratio $I_{\mathrm{D}} / I_{\mathrm{G}}$ for the $\mathrm{D}$ band and $\mathrm{G}$ band is widely used for characterizing the defect quantity in pristine and irradiated GO foils. Defects as well as oxygen functionalities in graphene significantly influence its transport and electrocatalytic properties. ${ }^{42}$ The $I_{\mathrm{D}} / I_{\mathrm{G}}$ ratio decreases with increasing ion fluence for the GO foils irradiated using $5.1 \mathrm{MeV}$ $\mathrm{He}^{2+}$ ions (see Table 5). This suggests that irradiation with $\mathrm{He}^{2+}$ ions leads to the formation of more $\mathrm{sp}^{2}$ graphene domains and this can be ascribed to the reduction of graphene oxide. ${ }^{27,36}$ This is also in agreement with XPS and ATR-FTIR results, which

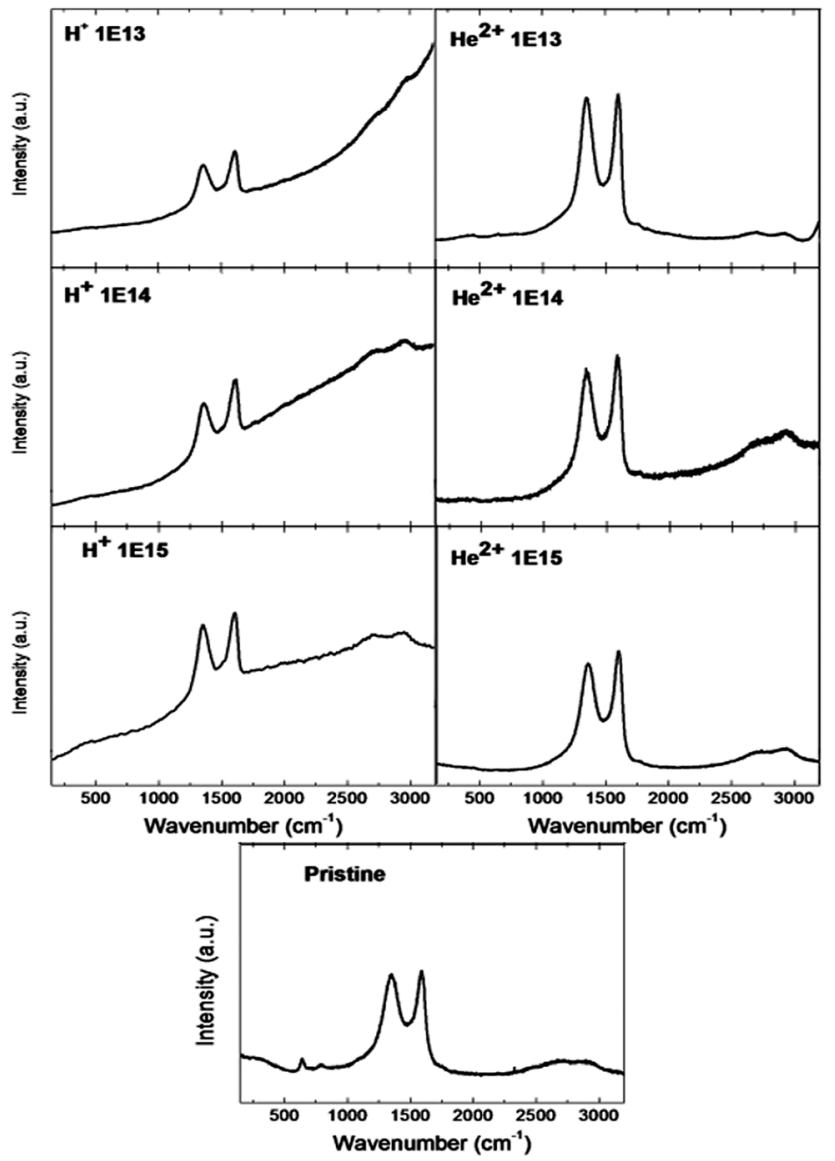

Fig. 6 The Raman spectra of pristine and irradiated GO foils. The ion beam irradiation was performed using $2.5 \mathrm{MeV} \mathrm{H}^{+}$and $5.1 \mathrm{MeV} \mathrm{He}^{+}$ions with different ion irradiation fluencies.
Table 5 The $D$ and $G$ peak intensity ratio from Raman spectra for pristine $\mathrm{GO}$ and for GO irradiated using $2.5 \mathrm{MeV} \mathrm{H}^{+}$and $5.1 \mathrm{MeV} \mathrm{He}^{+}$ions with different fluencies

\begin{tabular}{ll}
\hline Sample & $I_{\mathrm{D}} / I_{\mathrm{G}}$ \\
\hline Pristine graphene & 0.95 \\
$2.5 \mathrm{MeV} \mathrm{H}+$ & 0.91 \\
$2.5 \mathrm{MeV} \mathrm{H}{ }^{+} 1.0 \times 10^{13} \mathrm{~cm}^{-2} \mathrm{~cm}^{-2}$ & 0.91 \\
$2.5 \mathrm{MeV} \mathrm{H}^{+} 1.0 \times 10^{15} \mathrm{~cm}^{-2}$ & 1.08 \\
$5.1 \mathrm{MeV} \mathrm{He}^{2+} 1.0 \times 10^{13} \mathrm{~cm}^{-2}$ & 0.98 \\
$5.1 \mathrm{MeV} \mathrm{He}^{2+} 1.0 \times 10^{14} \mathrm{~cm}^{-2}$ & 0.90 \\
$5.1 \mathrm{MeV} \mathrm{He}^{2+} 1.0 \times 10^{15} \mathrm{~cm}^{-2}$ & 0.89
\end{tabular}

revealed carbon-carbon bond creation and destruction of oxygen functionalities along with oxygen release from the GO foil surface.

\section{Optical and electrical properties of the irradiated GO foils}

UV-Vis spectroscopy (UV-Vis) was used for the characterization of the structural changes of GO foil after ion irradiation. GO foils irradiated with $\mathrm{H}^{+}$and $\mathrm{He}^{2+}$ and also pristine foils were analysed by UV-Visible-IR spectroscopy over the range of fluencies from $1.0 \times 10^{13} \mathrm{~cm}^{-2}$ to $1.0 \times 10^{15} \mathrm{~cm}^{-2}$. Fig. 7A presents the normalized reflectivity curves for the virgin and the irradiated GO foils using a proton beam with $2.5 \mathrm{MeV}$ of energy. One can see the overlapping curves of all the investigated GO foils due to the very slight surface layer modification by $\mathrm{H}^{+}$ions. The reflectivity of GO foil irradiated by protons calculated in the visible wavelength region is about $2.1 \%$ for the pristine foil and ranges between 2.1 and $2.5 \%$ for the $2.5 \mathrm{MeV}$ $\mathrm{H}^{+}$irradiated GO foils. We didn't observe any significant influence of ion irradiation on UV-Vis reflectivity.

Fig. 7B shows the comparison between the reflectivity exhibited by the virgin and the ion irradiated GO foils using $\mathrm{He}^{2+}$ ions of 5.1 MeV. The graphene oxide foil irradiated using $\mathrm{He}^{2+}$ ions at a fluence of $1.0 \times 10^{15} \mathrm{~cm}^{-2}$ shows an increase in reflectivity compared to the other pristine and irradiated samples. This irradiated GO sample exhibits a reflectivity of about $4.2 \%$ in the visible wavelength region $(390-760 \mathrm{~nm})$ that corresponds to the photon energy in the range from 1.6 to $3.4 \mathrm{eV}$. This reflectivity enhancement can be connected to the band gap decline in the irradiated GO foil.

To investigate the change of electrical properties after ion irradiation, the current-voltage $(I-V)$ characteristics were measured using the standard two point method in the current range from $-1000 \mathrm{nA}$ to $1000 \mathrm{nA}$ (see Fig. 8). All pristine and irradiated GO foils exhibited a clear non-linear slope of the $I-V$ characteristic curve. After ion irradiation using an ion fluence of $1.0 \times 10^{15} \mathrm{~cm}^{-2}$, the GO foils showed a remarkable enhancement in current response compared to the pristine GO foil, for both ion species used. The electrical resistivity decrease is more pronounced for GO foil irradiated using 5.1 $\mathrm{MeV} \mathrm{He}^{+}$ions. These changes of the electrical resistivity after ion irradiation are in accordance with the creation of $\mathrm{sp}^{2}$ domains confirmed by Raman spectroscopy and the decrease of the oxygen concentration observed by XPS and RBS, simultaneously with the destruction of oxygen functional groups in the surface of irradiated GO foils as 

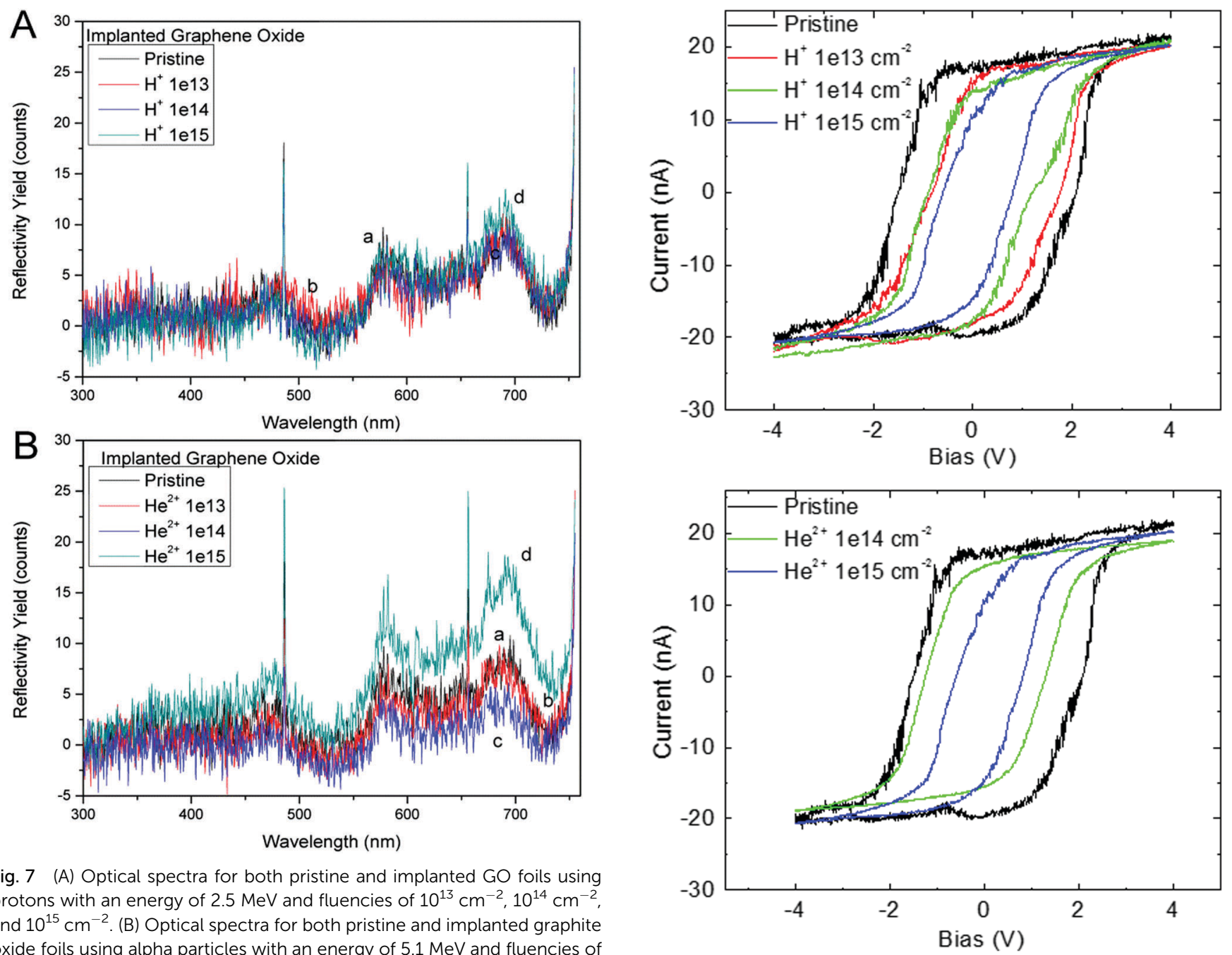

Fig. 7 (A) Optical spectra for both pristine and implanted GO foils using protons with an energy of $2.5 \mathrm{MeV}$ and fluencies of $10^{13} \mathrm{~cm}^{-2}, 10^{14} \mathrm{~cm}^{-2}$, and $10^{15} \mathrm{~cm}^{-2}$. (B) Optical spectra for both pristine and implanted graphite oxide foils using alpha particles with an energy of $5.1 \mathrm{MeV}$ and fluencies of $10^{13} \mathrm{~cm}^{-2}, 10^{14} \mathrm{~cm}^{-2}$, and $10^{15} \mathrm{~cm}^{-2}$.

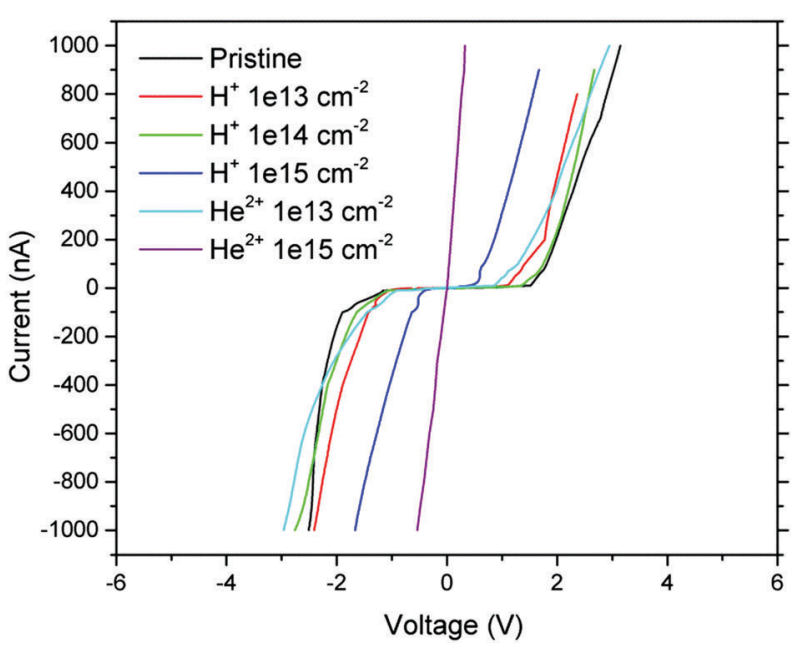

Fig. 8 The $I-V$ characteristics of pristine and irradiated GO foils obtained using the standard two point method. The ion beam irradiation was performed using $2.5 \mathrm{MeV} \mathrm{H}^{+}$and $5.1 \mathrm{MeV} \mathrm{He}^{2+}$ ions with different fluencies.
Fig. 9 The $I-V$ characteristics of pristine and irradiated $G O$ foils obtained using C-AFM. The ion beam irradiation was performed using $2.5 \mathrm{MeV} \mathrm{H}^{+}$ and $5.1 \mathrm{MeV} \mathrm{He}^{2+}$ ions with different fluencies.

observed by FTIR analysis. A conductive network in GO with many cross-linked carbon connections facilitates ion energy transfer to the graphene oxide structure via electronic stopping. ${ }^{36}$

Fig. 9 shows the cyclic $\mathrm{V}-\mathrm{A}$ graphs of pristine and irradiated GO foils measured by means of conductive AFM analysis (C-AFM). The voltage range during C-AFM analysis was in the range of $-4 \mathrm{~V}$ to $+4 \mathrm{~V}$. One can see clear non-linear characteristics and hysteresis around zero. The capacitive behaviour is decreasing with increasing ion fluence for both ion species used. This phenomenon can be connected to the created electrochemical active sites caused by deoxygenation and leads to the improvement in electrical conductivity. ${ }^{36}$

\section{Discussion}

Graphene is reported as a material with high electric conductivity, whereas graphene oxide is an electrical insulator and its reduction and deoxygenation can modify the electrical properties 
in such a way that GO can become a semiconductor or a conductor. $^{37,44}$ In our experiments, the GO foils irradiated by hydrogen and helium ions using the fluence $1.0 \times 10^{15} \mathrm{~cm}^{-2}$ exhibit much better conductivity compared to the pristine GO foil, and this tendency is more pronounced for the $\mathrm{He}^{2+}$ ions. This is in accordance with the results obtained by FTIR, XPS and Raman spectroscopy, which give us information that light ion irradiation of GO foils leads to a reduction of oxygen functionalities. Raman spectroscopy results suggest the formation of graphene $\mathrm{sp}^{2}$ domains after irradiation using $\mathrm{He}^{2+}$ ions. FTIR and XPS demonstrated that ion irradiation destroyed the surface oxygen chemical bonds $(\mathrm{C}=\mathrm{O}$ and $\mathrm{C}-\mathrm{O})$, and led to the formation of $\mathrm{C}=\mathrm{C}$ and $\mathrm{C}-\mathrm{C}$ bonds, whereas the significant oxygen release was not observed by RBS in deeper layers of GO samples. These changes of the chemical bonds, deoxygenation and carbonization of the GO surface, connected with an increase in conductivity, are caused by transfer of electronic energy during ion irradiation. ${ }^{31}$ The SRIM simulation shows that electronic stopping prevailing in the surface layer is higher for the $\mathrm{He}^{+}$ions compared to $\mathrm{H}^{+}$ones, which resulted in the more pronounced influence during irradiation using the $\mathrm{He}^{2+}$ ions. This is owing to the four times bigger mass of He compared to the $\mathrm{H}$. Moreover, the penetration of charged ions can enhance the electronic stopping in the target material. $^{31}$

\section{Conclusions}

The graphene oxide foils were irradiated using $2.5 \mathrm{MeV} \mathrm{H}^{+}$and $5.1 \mathrm{MeV} \mathrm{He}^{2+}$ ions with ion fluencies in the range of $1.0 \times$ $10^{13}-1.0 \times 10^{15} \mathrm{~cm}^{-2}$. C, O, and $\mathrm{H}$ contents in the bulk of pristine and irradiated GO foil obtained from RBS and ERDA analysis are similar for all samples, and thus, there seems to be no correlation between the ion irradiation fluence and the content of those elements. However, this is not surprising if we consider the highly prevalent electron stopping compared to the nuclear ones for the ion species used and their energy. On the other hand, the results of XPS, Raman and ATR-FTIR surface analysis revealed significant changes after ion irradiation. The Raman spectra and FTIR analysis showed the formation of $\mathrm{sp}^{2}$ bonded carbon atoms and growth of the graphene domains and reduction of the GO foil surface. From XPS and FTIR results it can be concluded that the electronic structure and chemical bonds in the irradiated GO foil surface are directly connected to the ion species and their fluence. In addition, the higher ion fluence caused the roughness decline and self-evident electrical resistivity decrease of the ion irradiated GO foils. The presented study shows that irradiation using light energetic ions is one of the methods for modification of graphene oxide that can change its optical and electrical properties.

\section{Acknowledgements}

The research has been carried out at the CANAM (Center of Accelerators and Nuclear Analytical Methods) infrastructure (LM2015056) and has been supported by Czech Science
Foundation (GACR No. 16-05167S). Z. S., J. L. and D. B. were supported by specific university research (MSMT No. 20-SVV/ 2017).

\section{Notes and references}

1 D. R. Dreyer, S. Park, Ch. W. Bielawski and R. S. Ruoff, Chem. Soc. Rev., 2010, 39, 228-240.

2 Y. P. Tang, D. R. Paul and T. S. Chung, J. Membr. Sci., 2014, 458, 199-208.

3 V. Mazánek, O. Jankovský, J. Luxa, D. Sedmidubský, Z. Janoušek, F. Šembera and Z. Sofer, Nanoscale, 2015, 7, 13646-13655.

4 G. Liu, W. Jin and N. Xu, Chem. Soc. Rev., 2015, 44, 5016-5030.

5 Q. Xu, H. Xu, J. Chen, Y. Lv, Ch. Dong and T. S. Sreeprasad, Inorg. Chem. Front., 2015, 2, 417-424.

6 Ch. Chi, X. Wang, Y. Peng, Y. Qian, Z. Hu, J. Dong and D. Zhao, Chem. Mater., 2016, 28, 2921-2927.

7 D. Jariwala, V. K. Sangwan, L. J. Lauhon, T. J. Marks and M. C. Hersam, Chem. Soc. Rev., 2013, 42, 2824-2860.

8 D. R. Dreyer, S. Park, Ch. W. Bielawski and R. S. Ruoff, Chem. Soc. Rev., 2010, 39, 228-240.

9 R. K. Joshi, S. Alwarappan, M. Yoshimura, V. Shajwala and Y. Nishima, Appl. Mater. Today, 2015, 1, 1-12.

10 Ch. K. Chua and M. Pumera, Chem. Soc. Rev., 2014, 43, 291-312.

11 Y. Zhu, S. Murali, W. Cai, X. Li, J. W. Suk, J. R. Potts and R. S. Ruoff, Adv. Mater., 2010, 22, 3906-3924.

12 M. Pumera, Electrochem. Commun., 2013, 36, 14-18.

13 G. Buchowicz, P. R. Stone, J. T. Robinson, C. D. Cress, J. W. Beeman and O. D. Dubon, Appl. Phys. Lett., 2011, 98, 032102.

14 J. H. Chen, W. G. Cullen, C. Jang, M. S. Fuhrer and E. D. Williams, Phys. Rev. Lett., 2015, 102, 236805.

15 B. D. Guo, Q. A. Liu, E. D. Chen, H. W. Zhu, L. A. Fang and J. R. Gong, Nano Lett., 2010, 10, 4975.

16 M. C. Lemme, D. C. Bell, J. R. Williams, L. A. Stern, B. W. H. Baugher, P. Jarillo-Herrero and C. M. Marcus, ACS Nano, 2009, 3, 2674.

17 M. Kalbac, O. Lehtinen, A. V. Krasheninnikov and J. Keinonen, Adv. Mater., 2015, 25, 1004-1009.

18 D. J. McComas, F. Allegrini, C. J. Pollock, H. O. Funsten, S. Ritzau and G. Gloeckler, Rev. Sci. Instrum., 2004, 75, 4863-4870.

19 M. Y. Han, B. Ozyilmaz, Y. Zhang and P. Kim, Phys. Rev. Lett., 2007, 98, 206805.

20 H. O. Funsten, F. Allegrini, P. Bochsler, G. Dunn, S. Ellis, D. Everett, M. J. Fagan, S. A. Fuselier, M. Granoff and M. Gruntman, et al., Space Sci. Rev., 2009, 146, 75-103.

21 M. Gruntman, Rev. Sci. Instrum., 1997, 68, 3617-3656.

22 D. Hovestadt, M. Hilchenbach, A. Bürgi, B. Klecker, P. Laeverenz, M. Scholer, H. Grünwaldt, W. I. Axford and S. Livi, et al., Sol. Phys., 1995, 162, 441-481.

23 Y. Zhang, L. Guo, S. Wei, Y. He, H. Xia, Q. Chen, H. B. Sun and F. S. Xiao, Nano Today, 2010, 5, 15-20. 
24 A. H. Castro Neto, F. Guinea, N. M. R. Peres, K. S. Novoselov and A. K. Geim, Rev. Mod. Phys., 2009, 81, 109.

25 V. M. Pereira, F. Guinea, J. M. B. Lopes dos Santos, N. M. R. Peresand and A. H. Castro Neto, Phys. Rev. Lett., 2006, 96, 036801.

26 R. Mikšová, A. Macková, P. Malinský and Z. Sofer, The stopping power and energy straggling of light ions in graphene oxide films, will be published.

27 O. Jankovský, P. Šimek, J. Luxa, D. Sedmidubský, I. Tomandl, A. Macková, R. Mikšová, P. Malinský, M. Pumera and Z. Sofer, ChemPlusChem, 2015, 80, 1399-1407.

28 Ch. K. Chua and M. Pumera, Small, 2015, 11, 1266-1272.

29 M. Mayer, SIMNRA version 6.06, Max-Planck-Institut fur Plasmaphysik Garching, Germany, 2006, available at: http:// www.rzg.mpg.de/ mam/.

30 G. Compagnini, F. Giannazzo, S. Sonde, V. Raineri and E. Rimini, Carbon, 2009, 47, 3201-3207.

31 V. Georgakilas, Functionalization of Graphene, John Wiley \& Sons, Germany, 2014, ISBN 352733551X.

32 Ch. K. Chua, A. Ambrosi and M. Pumera, Analyst, 2013, 138, 7012-7015.

33 J. F. Ziegler, et al., SRIM: The Stopping and Range of Ions in Matter, Version SRIM-2013, available at: http://www.srim.org/.
34 I. I. Bobrinetskiy, A. V. Emelianov, S. A. Smagulova, I. A. Komarov, N. Otero and P. M. Romero, Mater. Lett., 2017, 187, 20-23.

35 P. Kumar, K. S. Subrahmanyan and C. N. R. Rao, Int. J. Nanosci., 2011, 10, 559.

36 K. Hareesh, R. P. Joshi, B. Shateesh, K. Asokan, D. Kanjilal, D. J. Late, S. S. Dahiwale, V. N. Bhoraskar, S. K. Haram and S. D. Dhole, J. Phys. D: Appl. Phys., 2015, 48, 365105.

37 J. Chen, G. Zhang, B. Luo, D. Sun, X. Yan and Q. Xue, Carbon, 2011, 49, 3141-3147.

38 N. Cao and Y. Zhang, J. Nanomater., 2015, 2015, 16812.

39 L. Shahriary and A. A. Athawale, Int. J. Renew. Energy Environ. Eng., 2014, 2, 58-63.

40 O. Jankovský, P. Šimek, K. Klimová, D. Sedmidubský, S. Matějková, M. Pumera and Z. Sofer, Nanoscale, 2014, 6, 6065.

41 M. M. Viana, M. C. F. S. Lima, J. C. Forsythe, V. S. Gangoli, M. Cho, Y. Cheng, G. G. Silva, M. S. Wong and V. Caliman, J. Braz. Chem. Soc., 2015, 26, 978-984.

42 M. A. Pimenta, G. Dresselhaus, M. S. Dresselhaus, L. G. Cançado, A. Jorio and R. Saito, Phys. Chem. Chem. Phys., 2007, 9, 1276-1291.

43 E. D. Dikio, F. T. Thema, A. M. Farah and N. D. Shoto, Mater. Sci.-Pol., 2013, 31, 59-64.

44 S. Pei and H. M. Cheng, Carbon, 2012, 50, 3210-3228. 